\title{
Functional Chronic Constipation: Rome III Criteria Versus Rome IV Criteria
}

\author{
Marina Russo, ${ }^{1}$ Caterina Strisciuglio, ${ }^{2}$ Elena Scarpato, ${ }^{1}$ Dario Bruzzese, ${ }^{3}$ Marianna Casertano, ${ }^{2}$ and Annamaria Staiano ${ }^{1 *}$ \\ ${ }^{1}$ Department of Translational Medical Science, Section of Pediatrics, University of Naples "Federico II", Naples, Italy; ${ }^{2}$ Department of Women, \\ Child and Specialist Surgery, University of Campania "Luigi Vanvitelli", Naples, Italy; and ${ }^{3}$ Department of Public Health, University of Naples \\ "Federico II", Naples, Italy
}

\section{Background/Aims}

Functional constipation $(\mathrm{FC})$ is aa frequent functional gastrointestinal disorder, diagnosed according to the Rome criteria. In this study, we compared Rome III and Rome IV criteria for the diagnosis of FC, and determined the prevalence of FC according to these criteria.

\section{Methods}

Consecutive children between infancy and 17 years old were recruited for the study, excluding those with a known organic gastrointestinal disease. A prospective longitudinal design has beenused. For the diagnosis of FC, questionnaires on Pediatric Gastrointestinal Symptoms (QPGS) based on the Rome III and Rome IV criteria (QPGS-RIII and QPGS-RIV) were used. The agreement between these 2 questionnaires was measured by Cohen's kappa coefficient.

\section{Results}

Two hundred fourteen children (mean age, $77.4 \pm 59.5$ months; 103 males) were screened. There was no statistically significant difference in the prevalence of FC evaluated using the QPGS-Rome IV vs the QPGS-Rome III in the overall sample (39/214 [18.2\%] vs $37 / 214[17.3 .0 \%] ; P=0.831)$ as well as in any of the groups. The Cohen's kappa test showed a good agreement between the 2 criteria ( $\mathrm{\kappa}=0.65 ; 95 \% \mathrm{Cl}, 0.51$ to 0.78 ).

\section{Conclusion}

Our study demonstrates that the new Rome IV criteria have a good agreement with the Rome III criteria for the diagnosis of FC, without an increase in the number of potential diagnoses, despite the reduction in the duration of the symptoms. This conclusion is important in the management of childhood FC, since a late diagnosis negatively affects the prognosis.

(J Neurogastroenterol Motil 2019;25:123-128)

\section{Key Words}

Functional constipation; Pediatric; Questionnaires

Received: March 3, 2018 Revised: June 25, 2018 Accepted: October 9, 2018

() This is an Open Access article distributed under the terms of the Creative Commons Attribution Non-Commercial License (http://creativecommons. org/licenses/by-nc/4.0) which permits unrestricted non-commercial use, distribution, and reproduction in any medium, provided the original work is properly cited.

${ }^{*}$ Correspondence: Annamaria Staiano, MD

Department of Translational Medical Sciences, Section of Pediatrics, University of Naples, Federico II Via S. Pansini, 5, 80131 Naples, Italy

Tel: +39(0)817462679, Fax: +39(0)817462679, E-mail: staiano@unina.it

Marina Russo and Caterina Strisciuglio participated equally in this study. 


\section{Introduction}

Functional constipation (FC) is frequent in children. It is characterized by abdominal pain , evacuation of hard stool emission and reduced bowel movements.

The prevalence of pediatric FC ranges between 0.7-29.6\% and it has a high impact on healthcare costs. ${ }^{1,2}$ Symptoms often appear early in life. A study conducted by Malowitz et $\mathrm{al}^{3}$ has shown that the median age of onset of FC is 2.3 years. Constipation symptoms may lead to reduction in health-related quality of life, poor school performances and difficult social interactions at a time that the child is known to lay social and educational foundations for its future. ${ }^{4}$ Since FC is one of the most frequent diseases in very early age, it is essential to diagnose this functional disorder promptly

Perhaps, a delay in the diagnosis and in the treatment is negatively correlated with the recovery of childhood FC, therefore it is fundamental a correct diagnosis and a previous intervention. ${ }^{5}$

Currently, the diagnosis of FC is based on the new Rome IV criteria, ${ }^{6,7}$ which are the updated version of the Rome III criteria. ${ }^{8,9}$ Only minor changes have been made in the Rome IV diagnostic criteria compared to the previous Rome III criteria. ${ }^{10}$ In the group of neonate/toddlers, considering that the majority of toddlers aged less than 2.5 years are not toilet trained, a differentiation between toilet-trained and not toilet-trained children has been included. In fact, it is not possible to recognize fecal incontinence (FI) in a child wearing a diaper. In the group of child/adolescent the only modification is the decrease from 2 months to 1 month in the duration of symptoms needed to fulfill the criteria for the diagnosis. In accordance with the latest European and North American Societies for Pediatric Gastroenterology, Hepatology and Nutrition (ESPGHAN/NASPGHAN) constipation guidelines, ${ }^{11}$ a shorter duration of symptoms is needed for the definition of FC in the child/ adolescent group.

Our objective is to evaluate the agreement between Rome III and Rome IV criteria for the diagnosis of FC and the role of the related questionnaires in the evaluation of its prevalence.

\section{Materials and Methods}

The prevalence of $\mathrm{FC}$ was assessed based on the questions regarding defecation frequency ( $<2$ times per week), stool consistency, painful defecation, stool withholding behavior, large diameter stools and FI.

\section{Subjects}

Study subjects were consecutively recruited among children between infancy and 17 years old attending their general pediatrician for growth monitoring. Patients with a clinical history of organic gastrointestinal disease (eg, celiac disease, inflammatory bowel disease, food allergy, and surgery of the gastrointestinal tract) were excluded "a priori" and were not offered to participate in the study.

\section{Ethical Considerations}

Written informed consent was obtained from participants' parents, and the assent was obtained for all patients older than 10 years. The study was approved by the Institutional Review Board of the University of Naples "Federico II" (No. 58/18).

\section{Measures and Procedures}

The Questionnaire on Pediatric Gastrointestinal Symptoms (QPGS) is a validated instrument designed to classify gastrointestinal symptoms associated with functional gastrointestinal according to the Rome III and Rome IV criteria (QPGS-RIII and QPGSRIV). ${ }^{6-10}$ Each questionnaire is presented in 3 forms, due to the fact that diagnostic criteria are different for infants and toddlers and for children and adolescents: form A, for parents of infants and toddlers up to the age of 4 years; form $\mathrm{B}$, for parents of children and adolescents between 4 and 10 years of age; and form $\mathrm{C}$, for children and adolescents aged between 10 and 17 years. These 3 forms also collect sociodemographic and medical/developmental information.

Parents of all children younger than 10 years and adolescents aged 10 to 17 years old completed the age-related QPGS-RIII and QPGS-RIV helped by a research assistant. Compilation of both questionnaires took about 10 minutes.

The main characteristics of children's bowel habits were investigated: frequency of bowel movements, consistency of stools, onset of constipation symptoms, hard or painful bowel movements, family history of constipation, urgency or feeling of an unfinished bowel movement, mucus in stool, history of large-diameter stools that may block up the toilet, withholding stools for fear of pain, squeezing the legs or buttocks together (retentive posturing), FI (staining or soiling) during the day and/or night, large fecal mass in the rectum, and presence of associated symptoms.

All data were analyzed to establish how many patients met the Rome III, the Rome IV or both criteria.

\section{Statistical Methods}

Sample size was calculated separately with Epi-Info Statistical 
Calculator (Division of Health Informatics and Surveillance, Center for Surveillance, Epidemiology, and Laboratory Services, Clifton Road Atlanta, GA, USA) based on the estimated prevalence of functional constipation derived from the available literature, which is about $20 \%$. We considered a confidence interval (CI) of $95 \%$ and a precision error of $2 \%$. Assuming this, the sample size was 262 children between 0 and 17 years.

Numerical data are expressed as mean $\pm \mathrm{SD}$, while categorical variables were expressed as absolute frequencies and percentages.

Difference in prevalence of FC according to the 2 criteria was assessed using the McNemar test for paired samples and the agreement was globally measured using the Cohen's kappa coefficient $\kappa$ statistics (0-0.4, poor agreement; 0.4-0.6, good agreement; 0.6-0.8, very good agreement; $0.8-1$, excellent agreement) with the correspondent $95 \% \mathrm{CI}$.

Statistical analyses were performed using SPSS 22.0 (IBM Corporation, Armonk, NY, USA). A 2 -sided $P$-value $<0.05$ was considered statistically significant.

\section{Results}

Between January and May 2017, 220 children were consecutively screened. The parents of 6 children could not answer the questionnaires, therefore these patients were excluded from the study.

Clinical and demographic characteristics of the 214 children enrolled, are shown in Table 1 . No statistically significant differenc- es were found in the prevalence of FC estimated using the Rome III versus the Rome IV criteria: 37/214 (17.3\%) vs 39/214 (18.2\%), respectively $(P=0.831)$.

The Cohen's $\kappa$ test showed a good agreement between the Rome III and Rome IV criteria, for the definition of FC ( $\kappa=0.65$; 95\% CI, 0.51 to 0.78 ).

The distribution of FC in the 214 subjects according to the Rome III and Rome IV criteria, stratified for the 2 age groups, is shown in Table 2. In particular, in the sub-group $<4$ years, 11/81 children (13.6\%) fulfilled the Rome IV criteria while only $8 / 81$ subjects $(9.9 \%)$ fulfilled the Rome III criteria $(P=0.508)$. In the subgroup $>4$ years, $28 / 133$ children $(21.0 \%)$ fulfilled the Rome IV criteria while 29/133 subjects (21.8\%) fulfilled the Rome III criteria $(P>0.99)$.

Twenty-seven subjects out of 214 (12.6\%) fulfilled both the Rome III and the Rome IV criteria for FC, 10/214 (4.7\%) fulfilled only the Rome III, whereas $12 / 214$ were positive according to Rome IV criteria. These subjects were mainly from the group aged 4 to 17 years (Table 2 ).

The Cohen's $\kappa$ test between the 2 questionnaires regarding defecation frequency $\leq 2$ times/week, painful defecation, history of large diameter of stools, and FI showed a very good agreement ( 0.79 [0.61 to 0.97 ], 0.81 [0.73 to 0.9 ], 0.93 [0.83 to 1.03$], 0.61$ [0.35 to 0.87 ], respectively), while for hard stools the agreement was good (0.56 [0.41 to 0.7$])$.

Table 1. Demographical Characteristics of the Study Subjects According to Age Group

\begin{tabular}{lcccc}
\multicolumn{1}{c}{ Subjects } & All subject & 4 to $17 \mathrm{yr}$ & $<4 \mathrm{yr}$ & P-value \\
Number of subjects & 214 & 133 & 81 & $\mathrm{NA}$ \\
Age (mo) & $79.9 \pm 58.5$ & $113.8 \pm 43.8$ & $18.1 \pm 14.8$ & $\mathrm{NA}$ \\
Females & $111(51.9)$ & $75(56.4)$ & $36(44.4)$ & 0.590 \\
\hline
\end{tabular}

NA, not available.

Data are presented as mean $\pm \mathrm{SD}$ or $\mathrm{n}(\%)$.

Table 2. Distribution of the 214 Subjects According to Rome III and Rome IV Criteria, Stratified According to the Age Group

\begin{tabular}{cccc}
\hline \multirow{2}{*}{ Rome criteria } & \multicolumn{3}{c}{ Age group } \\
& $<4 \mathrm{yr}(\mathrm{n}=81)$ & $4-17 \mathrm{yr}(\mathrm{n}=133)$ & Total $(\mathrm{N}=214)$ \\
\cline { 2 - 4 } Rome III-/Rome IV- & $67(82.7)$ & $98(73.7)$ & $165(77.1)$ \\
Rome III-/Rome IV+ & $6(7.4)$ & $6(4.5)$ & $12(5.6)$ \\
Rome III+/Rome IV- & $3(3.7)$ & $7(5.3)$ & $10(4.7)$ \\
Rome III+/Rome IV+ & $5(6.2)$ & $22(16.5)$ & $27(12.6)$ \\
\hline
\end{tabular}

- , negative diagnosis; + , positive diagnosis

Data are presented as $\mathrm{n}(\%)$. 


\section{Subgroup Analysis}

\section{Infants and toddler group}

Looking at the clinical characteristics of the infants and toddler group (0-4 years old) we found that 2/81 (2.4\%) patients showed a bowel frequency $\leq 2$ times/week ( 1 according to Rome III and 1 according to Rome IV criteria). Regarding painful defecations, 23/81 (28.4\%) patients were identified (17 according to both Rome III and Rome IV criteria, 2 according to Rome III and 4 according to Rome IV questionnaire). Moreover 4/81 had large diameter stools according to both questionnaires. Hard stool were reported in 16/81 (19.7\%), 8/81 according to both questionnaires, 1 according to Rome III and 7 according Rome IV criteria (Table 3).

In this group of patients, 17/81 (21.0\%) had acquired toilet training skills at a median age of 27.6 months. FI was present in
4/17 (23.5\%): 1 according to both questionnaires, 3 according to Rome III criteria.

\section{Child and adolescent group}

In the child and adolescent group (4-17 years old), 10/133 (7.5\%) subjects showed a bowel frequency $\leq 2$ times/week according to both Rome III and Rome IV criteria, while 3 according to Rome III criteria. Forty-five/133 (33.8\%) children reported painful defecation (35 were identified according to Rome III and Rome IV criteria, 5 were positive to Rome III and 5 to Rome IV criteria). Moreover, 12/133 (9.0\%) had large diameter stools (10 according to both Rome III and Rome IV criteria, 1 according only to Rome III and 1 according to Rome IV criteria). Finally, 39/133 (29.3\%) reported hard stool, 18 according to both questionnaires, 13 according to Rome III and 8 according to Rome IV criteria (Table 3). FI was present in 9/133 (6.7\%) 5 according to both questionnaires, 3

Table 3. Agreement Between the Rome III and Rome IV Questionnaires Regarding Defecation Frequency, Painful Defecation, Large-diameter Stools, Hard Stools, and Fecal Incontinence

\begin{tabular}{|c|c|c|c|}
\hline \multirow{2}{*}{ Rome criteria } & \multicolumn{3}{|c|}{ Age group } \\
\hline & $<4 \operatorname{yr}(\mathrm{n}=81)$ & $4-17$ yr $(\mathrm{n}=133)$ & Total $(\mathrm{N}=214)$ \\
\hline \multicolumn{4}{|c|}{ Defecation frequency $\leq 2 / \mathrm{wk}$} \\
\hline Rome III-/Rome IV- & $79(97.5)$ & $120(90.2)$ & $199(93)$ \\
\hline Rome III-/Rome IV+ & $1(1.2)$ & $0(0.0)$ & $1(0.5)$ \\
\hline Rome III+/Rome IV- & $1(1.2)$ & $3(2.2)$ & $4(1.9)$ \\
\hline Rome III+/Rome IV+ & $0(0.0)$ & $10(7.5)$ & $10(4.7)$ \\
\hline \multicolumn{4}{|l|}{ Painful defecation } \\
\hline Rome III-/Rome IV- & $58(71.6)$ & $88(66.1)$ & $145(68.1)$ \\
\hline Rome III-/Rome IV+ & $4(4.9)$ & $5(3.8)$ & $9(4.2)$ \\
\hline Rome III+/Rome IV- & $2(2.5)$ & $5(3.8)$ & $7(3.3)$ \\
\hline Rome III+/Rome IV+ & $17(21.0)$ & $35(26.3)$ & $52(24.4)$ \\
\hline \multicolumn{4}{|l|}{ Large-diameter stools } \\
\hline Rome III-/Rome IV- & $77(95.1)$ & 121(91) & $198(92.5)$ \\
\hline Rome III-/Rome IV+ & $0(0.0)$ & $1(0.7)$ & $1(0.5)$ \\
\hline Rome III+/Rome IV- & $0(0.0)$ & $1(0.7)$ & $1(0.5)$ \\
\hline Rome III+/Rome IV+ & $4(4.5)$ & $10(7.5)$ & $14(6.5)$ \\
\hline \multicolumn{4}{|l|}{ Hard stools } \\
\hline Rome III-/Rome IV- & $65(80.2)$ & $94(70.7)$ & $159(74.3)$ \\
\hline Rome III-/Rome IV+ & $7(8.6)$ & $8(6.0)$ & $15(7.0)$ \\
\hline Rome III+/Rome IV- & $1(1.2)$ & $13(9.8)$ & $14(6.5)$ \\
\hline Rome III+/Rome IV + & $8(9.9)$ & $18(13.5)$ & $26(12.1)$ \\
\hline \multicolumn{4}{|l|}{ Fecal incontinence } \\
\hline Rome III-/Rome IV- & $13(76.4)$ & $124(93.2)$ & $137(91.3)$ \\
\hline Rome III-/Rome IV+ & $0(0.0)$ & $1(0.7)$ & $1(0.6)$ \\
\hline Rome III+/Rome IV- & $3(17.6)$ & $3(2.3)$ & $6(4.0)$ \\
\hline Rome III+/Rome IV+ & $1(5.8)$ & $5(3.8)$ & $6(4.0)$ \\
\hline
\end{tabular}

- , negative diagnosis; + , positive diagnosis.

Data are presented as $\mathrm{n}(\%)$. 
according to Rome III and 1 according Rome IV criteria.

\section{Discussion}

Our study shows good agreement between the Rome III and the Rome IV criteria for the definition of FC, despite the symptoms are observed for a shorter time. This is very important because it is well known that an earlier diagnosis improves the outcome of children with FC. In a study by Bongers et al, ${ }^{12}$ it has been demonstrated that a delay between the onset of symptoms and the first visit was one of the factors related to constipation's recurrence in adulthood. We showed that the reduction in the symptoms' duration did not increase the number of false positive diagnoses. Indeed, we found that the overall prevalence of constipation was $17.3 \%$ according to the Rome III criteria and $18.2 \%$ according to the Rome IV criteria.

A systematic review of the available literature reported that the global prevalence of childhood $\mathrm{FC}$ ranges from $0.7 \%$ to $30 \%$. According to this review Asian countries have a lower prevalence of constipation (median 10.8\%), ${ }^{1}$ compared to North America (16\%), Europe (19.2\%), and Oceania (19.7\%). The authors suggested that this discrepancy could be due to cultural, dietary, genetic, environmental, and socioeconomic conditions, and to the different healthcare systems. Although, there is also a lack of uniformity in the criteria used for the diagnosis, since not all of these studies adopted the Rome III criteria. In our study, we found a fair agreement between the Rome IV and the Rome III criteria in establishing the prevalence of FC.

Concerning the prevalence of functional gastrointestinal disorders in infants and toddlers, only limited studies using the Rome III criteria have been published. In these studies, ${ }^{11} \mathrm{FC}$ was more frequent in toddlers than in infants, according also to recent findings from a retrospective chart review study, which described that the median age of onset of FC in children was 2.3 years. ${ }^{13} \mathrm{We}$ found that in the sub-group $<4$ years, $11 / 81$ children $(13.6 \%)$ fulfilled the Rome IV criteria while only $8 / 81$ subjects ( $9.9 \%$ ) were positive according to Rome III criteria, unfortunately we do not have the prevalence of $\mathrm{FC}$ in the infants group.

In the Rome IV questionnaires, another change has been the introduction of the Bristol stool form scale for the assessment of stool consistency in the group of children and adolescents. Indeed, we found a different percentage of patients reporting hard stools according to Rome III and Rome IV questionnaires. However, this difference did not reach statistical significance regarding the prevalence of FC. Therefore, our results are in accordance with the study from Koppen et al, ${ }^{14}$ which demonstrated that the agreement between the Bristol stool form scale and the parental report for assessing the prevalence of $\mathrm{FC}$ is excellent. As the previous authors reported, we also demonstrated that the introduction of the Bristol stool form did not influence the evaluation of the prevalence of $\mathrm{FC}$. Indeed, the Rome criteria encompasses many elements, not only stool consistency. Indeed, the Rome III criteria for hard and painful stools is a combined criterion, and children fulfill these criteria if they have either hard stools or painful defecation. This decreases the impact of stool consistency alone in diagnosing FC.

Finally, in the Rome IV criteria a last differentiation has been made between children who are toilet trained and children who are not, expecting this to be important for the definition of FI. In our study, 20\% of children with an age between 0-4 years had acquired toilet training skills in accordance with previous literature. ${ }^{14} \mathrm{How}-$ ever, in this group of patients FI was reported only in 4 children, with a prevalence of $\mathrm{FI}$ of $23.5 \%$. This is in line with the observation that FI is reported only in $20 \%$ of children with FC treated in primary care.$^{15,16}$

The strength of our study is that we have recruited these children from a large general outpatient clinic sample, therefore an unselected population, and had an adequate number of cases to have a good statistical power and draw conclusions. Furthermore, we "diagnosed" FC not only on the basis of a questionnaire, but we also included a physical examination. In fact, a questionnaire is only a screening tool and the physician's assessment is always necessary to properly diagnose $\mathrm{FC}$.

In summary, this study highlights that the new Rome IV criteria has a good agreement with the Rome III criteria. We conclude that the new Rome IV criteria does not increase the number of false positive diagnoses of FC, despite the reduction in the symptoms duration. However, it may improve the outcome in the treatment of $\mathrm{FC}$ due to an earlier diagnosis.

\section{Financial support: None.}

\section{Conflicts of interest: None.}

Author contributions: Annamaria Staiano is the guarantor of the article: designed the research study, critically revised the manuscript, and approved the final version and the submission; Marina Russo and Caterina Strisciuglio performed data acquisition, wrote the first draft of the manuscript, and approved the final version of the paper; Elena Scarpato critically revised the manuscript and approved the final version of the paper; Dario Bruzzese analyzed the data, critically revised the manuscript, and approved the final 
version of the paper; and Marianna Casertano performed clinical assessment and data acquisition and approved the final version and the submission.

\section{References}

1. Mugie SM, Benninga MA, Di Lorenzo C. Epidemiology of constipation in children and adults: a systematic review. Best Prac Res Clin Gastroenterol 2011;25:3-18.

2. Rajindrajith S, Devanarayana NM. Constipation in children: novel insight into epidemiology, pathophysiology and management. J Neurogastroenterol Motil 2011;17:35-47.

3. Malowitz S, Green M, Karpinski A, Rosenberg A, Hyman PE. Age of onset of functional constipation. J Pediatr Gastroenterol Nutr 2016;62:600-602.

4. Bongers ME, van Dijk M, Benninga MA, Grootenhuis MA. Health related quality of life in children with constipation-associated fecal incontinence. J Pediatr 2009;154:749-753.

5. Chogle A, Saps M. Yield and cost of performing screening tests for constipation in children. Can J Gastroenterol 2013;27:e35-e38.

6. Benninga MA, Faure C, Hyman PE, St James Roberts I, Schechter NL, Nurko S. Childhood functional gastrointestinal disorders: neonate/ toddler. Gastroenterology 2016;150:1443-1445, e2.

7. Hyams JS, Di Lorenzo C, Saps M, Shulman RJ, Staiano A, van Tilburg M. Childhood functional gastrointestinal disorders: Child/Adolescent. Gastroenterology 2016;150:1456-1468.

8. Hyman PE, Milla PJ, Benninga MA, Davidson GP, Fleisher DF,
Taminiau J. Childhood functional gastrointestinal disorders: neonate/toddler. Gastroenterology 2006;130:1519-1526.

9. Rasquin A, Di Lorenzo C, Forbes D, et al. Childhood functional gastrointestinal disorders: child/adolescent. Gastroenterology 2006;130:15271537.

10. Schmulson MJ, Drossman DA. What is new in Rome IV. J Neurogastroenterol Motil 2017;23:151-163.

11. North American Society for Pediatric Gastroenterology, Hepatology and Nutrition. Evaluation and treatment of constipation in children: summary of updated recommendations of the North American Society for Pediatric Gastroenterology, Hepatology and Nutrition. J Pediatr Gastroenterol Nutr 2006;43:405-407.

12. Bongers ME, van Wijk MP, Reitsma JB, Benninga MA. Long-term prognosis for childhood constipation: clinical outcomes in adulthood. Pediatrics 2010;126:e156-e162.

13. Devanarayana NM, Rajindrajith S. Association between constipation and stressful life events in a cohort of Sri Lankan children and adolescents. J Trop Pediatr 2010;56:144-148.

14. Koppen I.JN, Velasco-Benitez CA, Benninga MA, Di Lorenzo C, Saps M. Using the bristol stool scale and parental report of stool consistency as part of the Rome III criteria for functional constipation in infants and toddlers. J Pediatr 2016;177:44-48, e1.

15. Schum TR, Kolb TM, McAuliffe TL, Simms MD, Underhill RL, Lewis M. Sequential acquisition of toilet-training skills: a descriptive study of gender and age differences in normal children. Pediatrics 2002;109:E48.

16. Loening-Baucke V. Prevalence rates for constipation and faecal and urinary incontinence. Arch Dis Child 2007;92:486-489. 